\title{
Equation-free detection and continuation of a Hopf bifurcation point in a particle model of pedestrian flow
}

\author{
Corradi, Olivier; Hjorth, Poul G.; Starke, Jens
}

Published in:

S I A M Journal on Applied Dynamical Systems

Link to article, DOI:

$10.1137 / 110854072$

Publication date:

2012

Document Version

Publisher's PDF, also known as Version of record

Link back to DTU Orbit

Citation (APA):

Corradi, O., Hjorth, P. G., \& Starke, J. (2012). Equation-free detection and continuation of a Hopf bifurcation point in a particle model of pedestrian flow. S I A M Journal on Applied Dynamical Systems, 11(3), 1007-1032. https://doi.org/10.1137/110854072

\section{General rights}

Copyright and moral rights for the publications made accessible in the public portal are retained by the authors and/or other copyright owners and it is a condition of accessing publications that users recognise and abide by the legal requirements associated with these rights.

- Users may download and print one copy of any publication from the public portal for the purpose of private study or research.

- You may not further distribute the material or use it for any profit-making activity or commercial gain

- You may freely distribute the URL identifying the publication in the public portal 


\title{
Equation-Free Detection and Continuation of a Hopf Bifurcation Point in a Particle Model of Pedestrian Flow*
}

\author{
Olivier Corradi ${ }^{\dagger}$, Poul G. Hjorth ${ }^{\dagger}$, and Jens Starke
}

\begin{abstract}
Using an equation-free analysis approach we identify a Hopf bifurcation point and perform a twoparameter continuation of the Hopf point for the macroscopic dynamical behavior of an interacting particle model. Due to the nature of systems with a moderate number of particles and noise, the quality of the available numerical information requires the use of very robust numerical algorithms for each of the building blocks of the equation-free methodology. As an example, we consider a particle model of a crowd of pedestrians where particles interact through pairwise "social forces." The pedestrians move along a corridor where they are constrained by the walls of the corridor, and two crowds are aiming, from opposite directions, to pass through a narrowing doorway perpendicular to the corridor. We focus our investigation on the collective behavior of the model. As the width of the doorway is increased, we observe an onset of oscillations of the net pedestrian flux through the doorway, described by a Hopf bifurcation. An equation-free continuation of the Hopf point in the two parameters, door width and ratio of the pedestrian velocities of the two crowds, is performed.
\end{abstract}

Key words. particle models, agent-based model, equation-free analysis, numerical bifurcation analysis, Hopf bifurcation, two-parameter continuation, collective behavior, self-organization, pattern formation, pedestrian dynamics

AMS subject classifications. $34,37,65,70,91$

DOI. $10.1137 / 110854072$

1. Introduction. The collective macroscopic behavior of systems consisting of many subsystems has been the focus of a large number of investigations. Prominent examples are the emergence of coherent light in lasers and pattern formation in fluid dynamics, chemistry, and biology [15], [14], [38]. In many cases, it is possible to derive analytically, e.g., via a center-manifold reduction, the macroscopic dynamics resulting from the microscopically defined systems and to further analyze this macroscopic dynamics in terms of, e.g., its bifurcation structure, in order to gain insight into the macroscopic behavior and its dependence on certain parameters. For other cases, such a derivation of an explicit macroscopic dynamical description is not yet known, or, due to, e.g., a discrete structure like in a particle model with a fairly large but for continuum approximations not sufficiently large number of particles [41], this might not even be possible. Such a situation is considered here in some detail, with the aim of obtaining insight into the macroscopic behavior of particle models with a finite number of particles for which no macroscopic dynamical equations are known.

Nevertheless, we consider a situation where we believe that a description in terms of macroscopic dynamics is meaningful, as suggested by numerical experiments. For this situation, the

\footnotetext{
${ }^{*}$ Received by the editors November 4, 2011; accepted for publication (in revised form) by D. Barkley May 17, 2012; published electronically September 5, 2012.

http://www.siam.org/journals/siads/11-3/85407.html

${ }^{\dagger}$ Department of Mathematics, Technical University of Denmark, DK-2800 Kongens Lyngby, Denmark (olivier. corradi@gmail.com, p.g.hjorth@mat.dtu.dk, j.starke@mat.dtu.dk).
}

1007

Copyright (C) by SIAM. Unauthorized reproduction of this article is prohibited. 
equation-free, or coarse analysis, approach [43] provides a framework in which to perform, e.g., numerical bifurcation analysis for macroscopic dynamics which is not explicitly known but can be accessed via suitably chosen short microscopic model evaluations (for further details see, e.g., [43], [12], [9], [25], [26], [27]). Such a multiscale approach requires well-separated spatial and temporal scales between the macroscopic observables and the microscopic processes, respectively. In the system considered here, this means the individual particle motion evolves on a much faster time scale than the evolution of the macroscopic observables investigated, where averaged quantities like the center of mass have been chosen.

Even though the methods we use and develop are applicable to many different particle models, we specifically consider a model for describing pedestrian flow through a corridor with a bottleneck. The example of pedestrian models has drawn attention due to its relevance in emergency escape routes and other panic situations in huge crowds (see, e.g., [18]). It should be remarked that these models are quite close to physical particle models but in addition contain an overlap to social sciences where they can be regarded as relevant to quantify the social science [17].

Collective macroscopic behavior in a crowd of pedestrians moving along a hallway can be reproduced by simple dynamical models of a collection of mass points moving in a twodimensional domain under the influence of mutual interactions (particle-particle interactions or "social forces") as well as "constraint forces" from the walls. The self-organized emergence of structure, i.e., the pattern formation or the macroscopic behavior of pedestrian models, was already reported in [21] and subsequently in [18], [17], [19], [20], [24], [31], [39]. Recently the investigation of pedestrian models has also drawn the attention of the mathematical community; see, e.g., [3], [32], [2], [5], [6]. In the following we will focus on the emergence of oscillatory patterns in microscopic particle models for pedestrian flow as in [21]. Nevertheless, a more rigorous numerical analysis, with a deeper understanding of how the macroscopic behavior depends on certain parameters, has been missing so far for the oscillatory phenomena. This is what we aim to contribute with the present paper.

In the context of pedestrian modeling, the particle-particle "forces" are termed "social forces," as they model the social behavior of each pedestrian as this pedestrian moves along in a crowd of other pedestrians. Note that these are not forces in the physical sense but a cause for each pedestrian to adapt its own movements based on psychological interactions. Each pedestrian aims to move in a specific direction, so there is a force term attracting the pedestrian to a distant point (target). Each pedestrian has a "private space" and consequently moves to avoid too close proximity to other pedestrians. As long as this "private space" is not threatened, each pedestrian is indifferent to the configuration of the other pedestrians. In other words, our model does not include enochlophobic or agoraphobic behavior.

Observations [18], [11] suggest that the behavior of a real crowd strongly depends on the density of the crowd as well as on the constraints imposed, e.g., by walls or doorways. Also in the swarm dynamics investigated in [23], density plays a decisive role for a phase transition between ordered and disordered states. For low pedestrian densities, the single particle behavior dominates, altered only by well-separated particle interactions. For high pedestrian densities, the behavior of the system becomes strongly correlated, and under certain conditions one can observe an onset of collective modes: oscillations in net flux, and formation of lanes. For large enough densities, it is interesting to model, and attempt to quantify, the

Copyright (c) by SIAM. Unauthorized reproduction of this article is prohibited. 
transition between various macroscopic behaviors due to changes of system parameters. In particular we find in this work that for two counterstreaming populations simultaneously passing through a doorway from opposite directions, widening or narrowing the width of the doorway can trigger or terminate oscillations of particle flux, so that the two opposing populations take turns in letting one direction dominate in the doorway. We find the transition from the "jammed" state to the "oscillatory" state to be characterized by a Hopf bifurcation (see, e.g., [33], [16]).

In section 2 we define and discuss our pedestrian model and in more detail the functional form [35], [1], [42] of the "forces" employed. These finite range forces are more realistic than those suggested in [21]. Section 3 describes the types of self-organized macroscopic behavior observed in real crowds and simulations, as well as defines our macroscopic observables. In section 4 we investigate the domains of qualitatively different macroscopic behavior. We identify a Hopf bifurcation point numerically by varying the door width $w$. Next, we investigate a two-parameter space spanned by the width $w$ of the doorway and a relative "pressure" parameter $r_{v^{0}}$ measuring the ratio of the pedestrian velocities $v_{0}$ of the two pedestrian crowds. A value $r_{v^{0}}=1$ describes two similarly energetic crowds, while $r_{v^{0}}>1$ or $r_{v^{0}}<1$ models the case where one crowd is more energetic than the other.

These parameter regions are characterized by either a stationary or an oscillatory behavior and are separated by a line of Hopf bifurcation points. First, we detect the Hopf bifurcation point for $r_{v^{0}}=1$ by an equation-free analysis of the system by varying the door width $w$. Second, we perform an equation-free two-parameter continuation of the Hopf bifurcation point by varying both the door width $w$ and the relative pressure parameter $r_{v^{0}}$.

2. A particle model for pedestrian behavior. As mentioned in the introduction, we model the pedestrians as interacting mass points and describe their change of positions with coupled differential equations. In choosing the analytical form of the force terms governing the dynamics of each individual pedestrian, we attempt to compromise between realism and numerical efficiency. A slightly different version of the model suggested by Helbing and Molnár [21] has been used, where the angle of sight has been omitted, the pedestrian-pedestrian as well as the pedestrian-wall interaction has been changed to be of finite range, and the pedestrianpedestrian interaction has been made isotropic instead of anisotropic. The omission of the angle of sight and the use of the isotropic pedestrian-pedestrian interaction are simplifications in order to study the macroscopic phenomena in a minimalist model, whereas finite range forces make the model more realistic.

Here, the undisturbed motion of a single pedestrian $i$ is a motion that accelerates until its velocity $v_{i}(t)=\left\|\dot{\mathbf{x}}_{i}(t)\right\|$ reaches a fixed cruising velocity $v^{0}$. This can be obtained by subjecting each pedestrian to the velocity dependent "target force"

$$
\mathbf{F}_{i}^{(0)}=\frac{1}{\tau}\left(v^{0} \mathbf{e}_{i}^{0}-\dot{\mathbf{x}}_{i}(t)\right)
$$

containing a desired direction of motion $\mathbf{e}^{0}$. This desired direction of motion $\mathbf{e}^{0}=\mathbf{e}^{0}(t, \mathbf{x}(t))$ will in general depend on time $t$ and the current position $\mathbf{x}(t)$ of the pedestrian. In the pedestrian problem considered here, the choice of the desired direction of motion is as follows: Before the pedestrian has passed through the door, the centerpoint of the doorway is the

Copyright $\odot$ by SIAM. Unauthorized reproduction of this article is prohibited. 


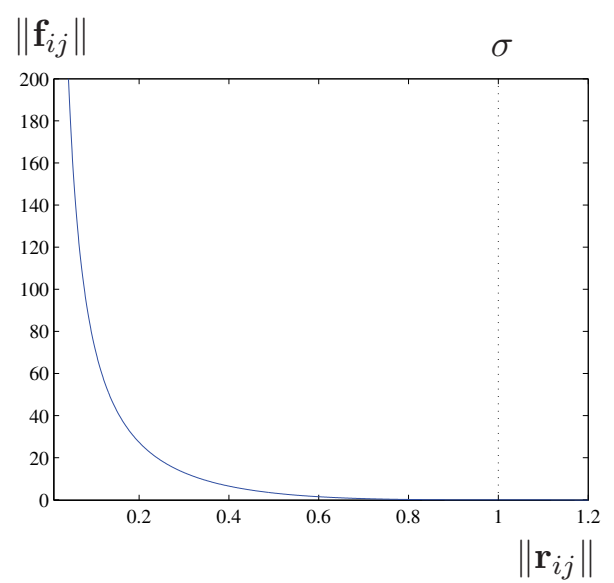

Figure 1. The magnitude $\left\|\mathbf{f}_{i j}\right\|$ of the pedestrian-pedestrian interaction force as a function of the particle distance $\left\|\mathbf{r}_{i j}\right\|$. The force is repelling, rising sharply as the separation becomes small, but also vanishes when the separation exceeds a value $\sigma$. In the simulations, we have used $V_{i j}=15, \sigma=1$. See Table 1 .

target. After the pedestrian has passed the door, the target shifts to a point at the end of the hallway which would cause an undisturbed pedestrian to move parallel to the corridor walls.

Next is the "social" or "territorial" force $f_{i j}$ between two individual pedestrians $i$ and $j$. Along the vector $\mathbf{r}_{i j}$ connecting two pedestrians, we take this similarly as in [42] to be a $C^{1}$ repelling force of finite range $\sigma$ (i.e., pedestrians with a distance larger than $\sigma$ away from each other do not interact):

$$
\mathbf{f}_{i j}= \begin{cases}-V_{i j}\left[\tan \left(\frac{\pi}{2}\left(\frac{\left\|\mathbf{r}_{i j}\right\|}{\sigma}-1\right)\right)-\frac{\pi}{2}\left(\frac{\left\|\mathbf{r}_{i j}\right\|}{\sigma}-1\right)\right] \frac{\mathbf{r}_{i j}}{\left\|\mathbf{r}_{i j}\right\|}, & \left\|\mathbf{r}_{i j}\right\| \leq \sigma, \\ \mathbf{0}, & \left\|\mathbf{r}_{i j}\right\|>\sigma,\end{cases}
$$

where the interaction strength is $V_{i j} \in \mathbb{R}$ and $\|\cdot\|$ is the Euclidean norm (Figure 1). The used finite range interactions are more realistic compared to those used in [21] because real pedestrians typically ignore other pedestrians sufficiently far away in their choice of walking direction. Note that in order to take into account the separating nature of walls, this force is set to zero if the wall containing the door separates two pedestrians.

In addition to this, we surround the walls and the doorway with a repelling potential that pushes pedestrians away from the nearest point of a wall $B$ with a force $\mathbf{f}_{i, B}$ that depends on the distance between pedestrians and this point. This force is chosen as (2) but weaker, i.e., with a smaller prefactor $U_{i, B}$ but with a larger characteristic distance $R$ than the pedestrianpedestrian interaction, i.e., with $R>\sigma$.

The motion of each pedestrian $i$ is then computed as the dynamics of a point mass particle in response to the prescribed sum of all "forces" (as in Figure 2) with the equation

$$
\ddot{\mathbf{x}}_{i}=\mathbf{F}_{i}^{0}+\sum_{j} \mathbf{f}_{i j}+\sum_{B} \mathbf{f}_{i, B}
$$

where $\mathbf{x}=(x, y), x$ being the coordinate along the corridor in an orthonormal coordinate system with the origin at the center of the door (see Figure 3). To solve this equation of

Copyright ( $\odot$ by SIAM. Unauthorized reproduction of this article is prohibited. 


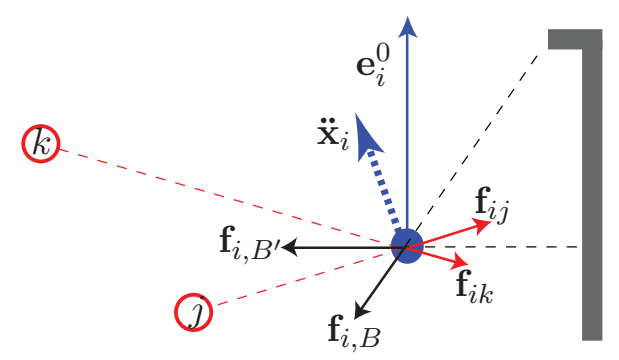

Figure 2. The behavioral "forces" between a pedestrian and nearby walls, as well as other pedestrians, the pedestrian's attraction towards a target location, and the resulting acceleration force $\ddot{\mathbf{x}}_{i}$.

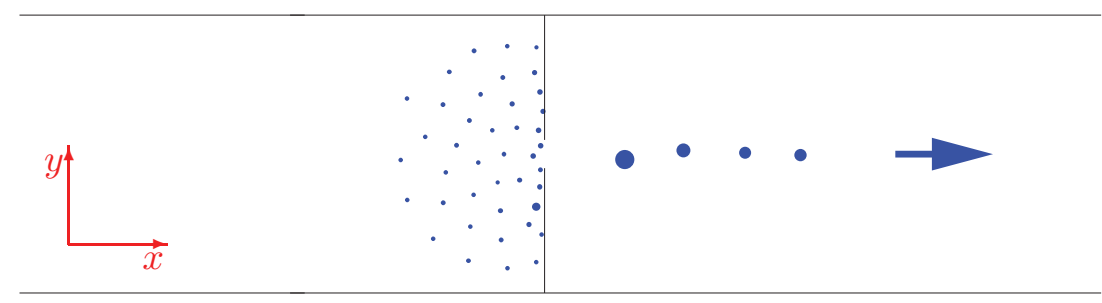

Figure 3. Crowd of pedestrians crowding in front of and passing through a bottleneck doorway. The radii of the circles are proportional to the magnitude of pedestrian velocities. See Table 1 for parameter values.

motion numerically, we rewrite it in the usual manner as a first order system with the state variables $(\mathbf{x}, \mathbf{v})$ with $\mathbf{v}=\dot{\mathbf{x}}$. To also model real pedestrian behavior in blocking situations where pedestrians try to step aside to escape pedestrian-pedestrian blocking, a noise term $\mathbf{n}_{i}$ is added to the velocity vector $\mathbf{v}$. This resulting equation of Langevin type is then solved numerically by an Euler method with step size $\Delta t$ where the noise term is scaled with $\sqrt{\Delta t}$ (see, e.g., [22]). Throughout the paper, we used a step size $\Delta t=0.001$.

The noise term $\mathbf{n}_{i}$ has two components $n_{i}^{\|}$and $n_{i}^{\perp}$, parallel and perpendicular (respectively) to the desired motion vector $\mathbf{e}_{i}$. Hereby, $n_{i}^{\|}$is normally distributed with mean zero and standard deviation $s_{\|}=0.00158$, and $n_{i}^{\perp}$ is normally distributed with mean 0.00632 and standard deviation $s_{\perp}=0.0632$. This mimics the tendency of particles to move to the right in case of a frontal collision. Introducing $\mathbf{e}_{i}^{\perp}$ as the vector orthogonal to the direction of motion with the right-hand rule, the noise term is expressed as

$$
\mathbf{n}_{i}=n_{i}^{\|} \mathbf{e}_{i}+n_{i}^{\perp} \mathbf{e}_{i}^{\perp}=n_{i}^{\|} \mathbf{e}_{i}+n_{i}^{\perp}\left(\begin{array}{cc}
0 & -1 \\
1 & 0
\end{array}\right) \mathbf{e}_{i} .
$$

The numerical simulations have been carried out using the parameters defined in Table 1. Note that we interpret the distance and time units as meters and seconds, respectively, because these are fairly natural length and time scales of the problem we are interested in. In order that the simulation does not run out of pedestrians as they reach the end of the hallway, we use periodic boundary conditions in $x$ and reinject the pedestrians who stream out at the end with some $y$ value at a new and random value of $y$ at the opposite end of the corridor. This is necessary, as to be able to observe a meaningful system behavior, the object of interest 
Table 1

Parameter values of the pedestrian model used for the simulations and numerical analysis.

\begin{tabular}{|l|l|}
\hline Total number of pedestrians & 200 \\
\hline Pedestrian terminal velocity & $v^{0}=1.5 \mathrm{~ms}^{-1}$ \\
\hline Acceleration relaxation time & $\tau=0.22 \mathrm{~s}$ \\
\hline Pedestrian-pedestrian interaction strength & $V_{i j}=15 \mathrm{~m}^{2} \mathrm{~s}^{-2}$ \\
\hline Pedestrian-pedestrian length scale & $\sigma=1 \mathrm{~m}$ \\
\hline Pedestrian-wall interaction strength & $U_{i, w}=10 \mathrm{~m}^{2} \mathrm{~s}^{-2}$ \\
\hline Pedestrian-wall length scale & $R=2 \mathrm{~m}$ \\
\hline Corridor width & $C_{w}=5 \mathrm{~m}$ \\
\hline Corridor length & $C_{l}=45 \mathrm{~m}$ \\
\hline
\end{tabular}

has to be an element of the $\omega$-limit set which describes the asymptotic behavior (see, e.g., [13]).

The focus of this study is to investigate the emergence of oscillatory patterns arising when two species are passing a narrow door in opposite directions. Later, in section 4, we will investigate systems where particle species on each side of the door have different values for $v^{0}$. This is described by the parameter $r_{v^{0}}$ in the model. This parameter describes a nonequal energy in the two particle populations, initially located on each side of the partition. Modeling two different pedestrian species $\alpha$ and $\beta$, each aiming to get through the doorway from opposite sides and towards the far end of the corridor, a variation in $r_{v^{0}}$ would make one population of pedestrians more vigorous in pushing its way through the other population. Specifically, we define $r_{v^{0}}$ as

$$
r_{v^{0}}=v_{\alpha}^{0} / v_{\beta}^{0},
$$

i.e., as the ratio of terminal velocities between the two species $\alpha$ and $\beta$ used in the equations of motion (3) with (1) and (2).

3. Observation of collective dynamics. Collective behavior, involving many individuals, can be observed both in real crowds [18], [11] and in computer simulations [21].

In the considered model, the individual, or microscopic, particles move according to very simple laws, being attracted to a distant point and repelled locally by other particles and by walls. We are working under the assumption that the boundary conditions have no influence on the macroscopic dynamics we are interested in. The validity of these assumptions is checked later in this section. The geometry of the considered model is simple, being characterized by only one parameter, the dimensionless width $w$ of the doorway.

For certain values of the door width $w$ we observe macroscopic oscillations which are shown in Figure 4. In order to quantify the observation of temporal changes in the number of particles passing the doorway, we introduce a variable being a measure for the overall position of a group of particles of a specific species. More specifically, we use a weighted center of mass

Copyright (c) by SIAM. Unauthorized reproduction of this article is prohibited. 


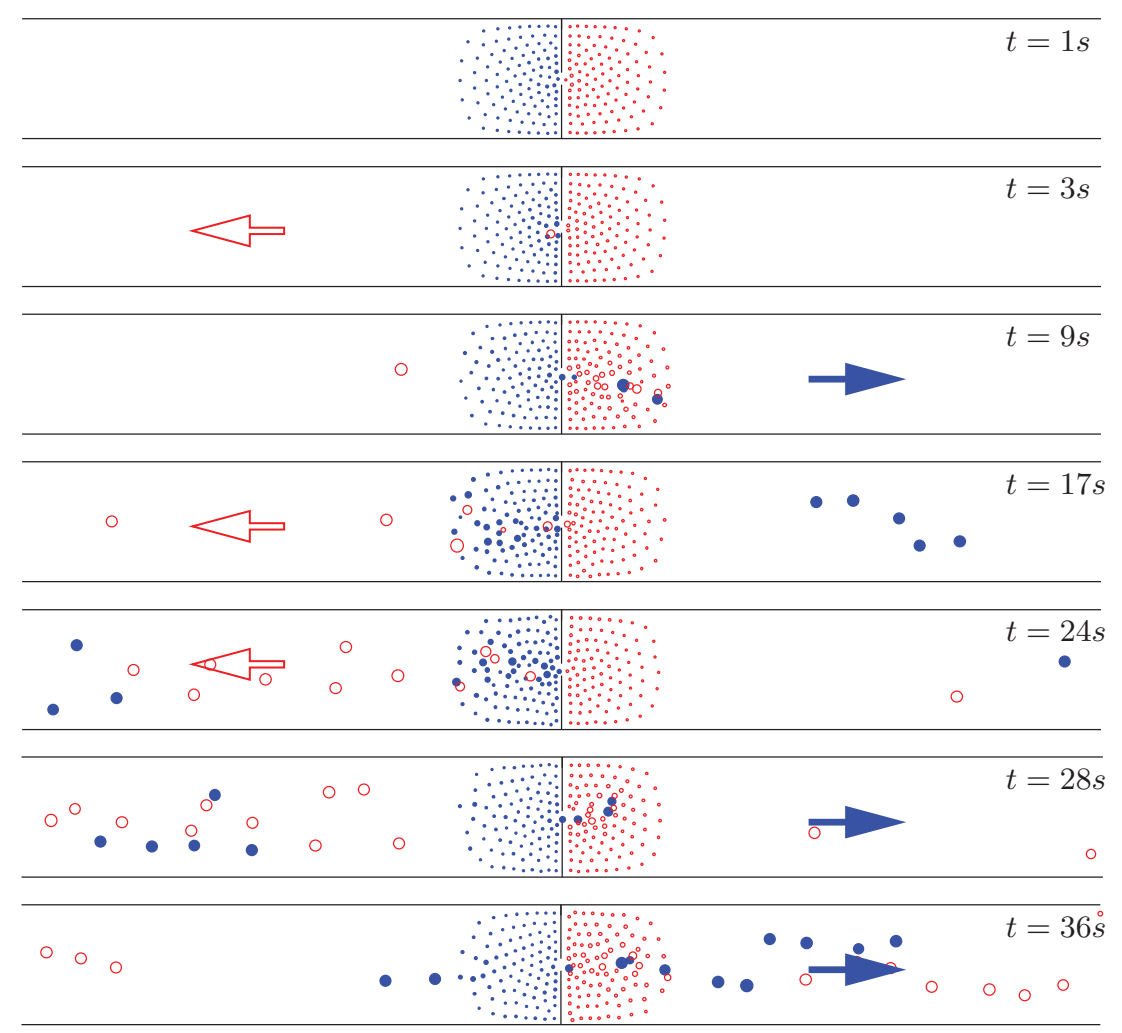

Figure 4. Snapshots from the oscillatory particle flow through the doorway. The arrows indicate the sign of the variable $\dot{m}$, the overall center of mass velocity. Notice in the final frame the phenomenon of the formation of a single lane of pedestrians emerging through the door, consistent with the observation as in [21]. The radii of the circles are proportional to the magnitude of pedestrian velocities. See Table 1 for parameter values.

variable $m$, such that, e.g., for the species $\alpha$,

$$
m_{\alpha}=\frac{\sum_{i \in \alpha} \kappa\left(x_{i}\right) x_{i}}{\sum_{i \in \alpha} \kappa\left(x_{i}\right)},
$$

where we consider only the longitudinal component $x_{i}$ (i.e., the component in the walking direction) using a coordinate system with the origin located in the center of the door. Note that $m_{\alpha}$ can remain constant even if particles are moving; two particles displacing simultaneously in opposite directions gives no change in $m_{\alpha}$. Dividing by the sum of weights keeps the measured quantity in the original unit.

The weight function $\kappa\left(x_{i}\right)$ is introduced to focus mainly on the particles that are positioned around the door. This permits us to separate the dynamics of the interactions at the door from the dynamics of particles traveling towards or away from it, and in particular discontinuously entering or leaving the corridor at the ends. By placing the origin of the coordinate system at the center of the door, the orthogonal distance of a particle $i$ to the door is then denoted 
with $\left|x_{i}\right|$. The weight function has the following characteristics:

$$
\kappa\left(x_{i}\right)= \begin{cases}1 & \text { for }\left|x_{i}\right| \leq d, \\ g\left(x_{i}\right) & \text { for } d \leq\left|x_{i}\right| \leq b, \\ 0 & \text { for }\left|x_{i}\right| \geq b\end{cases}
$$

where $d$ defines the size of the neighborhood around the door with maximal weighting and $\pm b$ bound the area taken into account. In our case, the bound $b$ is chosen as the corridor bound $C_{l} / 2$.

A cubic spline $g\left(x_{i}\right)$, i.e., a third order polynomial, is then used to strictly monotonously connect the two constant parts of the weight function. $C^{2}$ continuity of $\kappa(x)$ is ensured by imposing continuity of the first and second derivatives at the connection points, which is visualized in Figure 5.

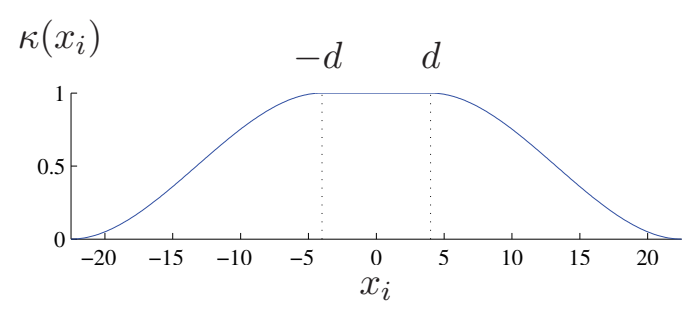

Figure 5. A weight function $\kappa\left(x_{i}\right)$, constructed from a cubic spline connecting two constant functions, is used to exclude the contribution of particles far away from the door. In our simulations the value $d=4$ with bound $b=C_{l} / 2=22.5$ is used.

Finally, as a compromise between the clarity of the macroscopic result and the smoothness of the macroscopic quantities in phase space introduced later, the center of mass of the entire population is computed as an average over the two species $\alpha$ and $\beta$ :

$$
m=\frac{1}{2}\left(m_{\alpha}+m_{\beta}\right) .
$$

The difference of $m_{\alpha}$ and $m_{\beta}$ would amplify what is going on macroscopically but would be worse in terms of the smoothness of the trajectory. The used mean between $m_{\alpha}$ and $m_{\beta}$ has a weaker but still clear signal of what is macroscopically going on and shows smoother trajectories.

We also wish to investigate temporal changes of $m$. The dynamics of the system are then explored as they unfold in the two-dimensional phase space with variables $(m, \dot{m})$. In the simulations, $\dot{m}$ is computed numerically using central differences $\left(m\left(t+\frac{\Delta t}{2}\right)-m\left(t-\frac{\Delta t}{2}\right)\right) / \Delta t$ with $\Delta t=0.1 \mathrm{~s}$. The change of the macroscopic dynamics is described with $(m, \dot{m})$, as shown in Figure 6. This makes sense because in a study of a one-dimensional oscillation in the $x$-direction of our coordinate system it is natural to introduce these two variables. This is also the easiest choice we could think of that represents the behavior in a transparent way. To have a periodic solution, i.e., a closed orbit in phase space, one needs a minimum of two dimensions. This dynamical system evolves according to a set of equations

$$
\frac{d}{d t}\left(\begin{array}{c}
m \\
\dot{m}
\end{array}\right)=F\left(\left(\begin{array}{c}
m \\
\dot{m}
\end{array}\right) ; w, r_{v^{0}}\right)
$$

Copyright $\odot$ by SIAM. Unauthorized reproduction of this article is prohibited. 
where the function $F$ on the right-hand side depends on the parameters $w$ and $r_{v^{0}}$, and is not explicitly known. First, we concentrate on the effect of the door width parameter $w$.

The range of the parameter $w$ for the door width can be set in the simulations to any value between $w=0$ (fully closed) and $w=C_{w}=5$ (fully open). For small values of the door width, the door is effectively blocking. Small oscillations in the particle flux, set up by the initial conditions, quickly dampen to zero, as shown in Figure 6(a). To sustain an oscillation of particle flux, as seen in Figure 6(c), larger values of $w$ are needed. Between these values, a later-identified supercritical Hopf bifurcation marks the transition from a stable fixed point to an unstable fixed point surrounded by a stable limit cycle.

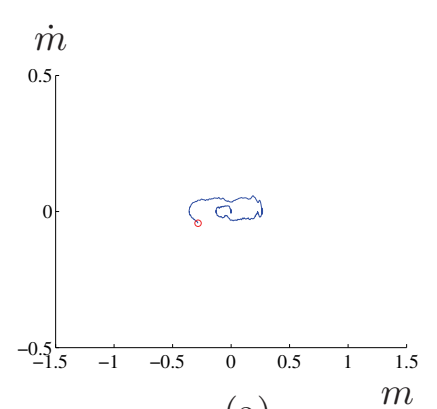

(a)

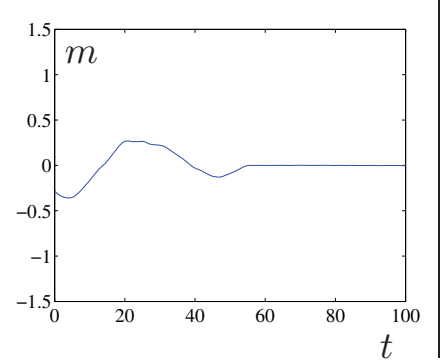

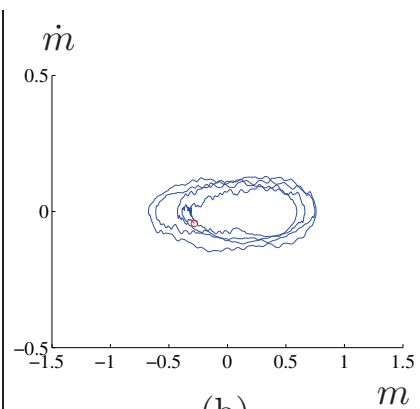

(b)

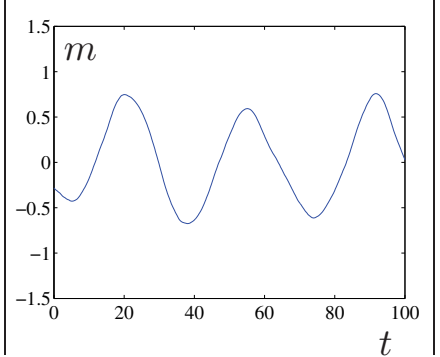

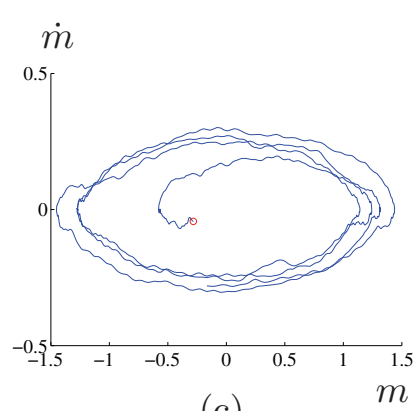

(c)

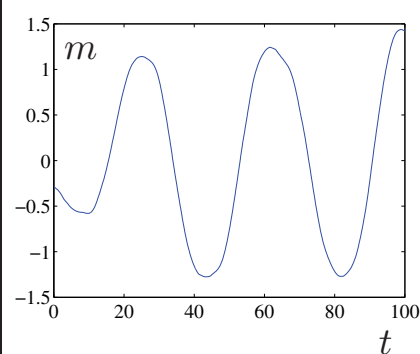

Figure 6. Depiction of the three types of qualitative behavior in phase space in the neighborhood of the onset of the observed oscillatory behavior. Each column shows a trajectory in the phase space ( $m, \dot{m})$ as well as $m(t)$ over time $t$ for a certain door width $w$. The red circle in the phase space marks the initial condition. Observe the different qualitative behavior showing the presence of a bifurcation. The corresponding bifurcation point will be investigated in section 4.1. In (a), before the bifurcation point, the phase space trajectory moves in towards the stable stationary point, and the time series for $m$ is a decaying oscillation. At $w=0.58$, just after the Hopf bifurcation, in (b) the stationary point is now unstable and is surrounded by a stable limit cycle. Finally, in (c) we show at $w=0.65$ behavior spiraling away from the unstable fixed point towards the stable limit cycle.

An obvious concern with the described system setup yielding periodic solutions is that the periodic boundary conditions could influence the dynamics and become a cause of observed collective phenomena. In particular we must ensure that there are no resonant effects of particle reinjection. The time scale (i.e., the period) associated with collective oscillatory modes (see section 4) is about 40 seconds, as seen in, e.g., Figure 6. The mean travel time $\langle\Theta\rangle_{\tilde{\alpha}}$ of particles from a group $\tilde{\alpha}$ is several minutes, as seen in Figure 7. Here, $\tilde{\alpha}$ represents only those particles that have completed a travel cycle in the observation time. The mean of the travel times is computed only for those pedestrians who traveled a full cycle during the 
observed time. There is no simple integer ratio of the two and the ratio changes continuously through a range of values, so that one can assume that the oscillatory behavior results from a self-organizing effect and is not due to an influence from the periodic boundary conditions. In addition to this, the collision avoidance and mixing through the doorway crowd spreads the distribution of travel times and hence further suppresses potential resonant effects.

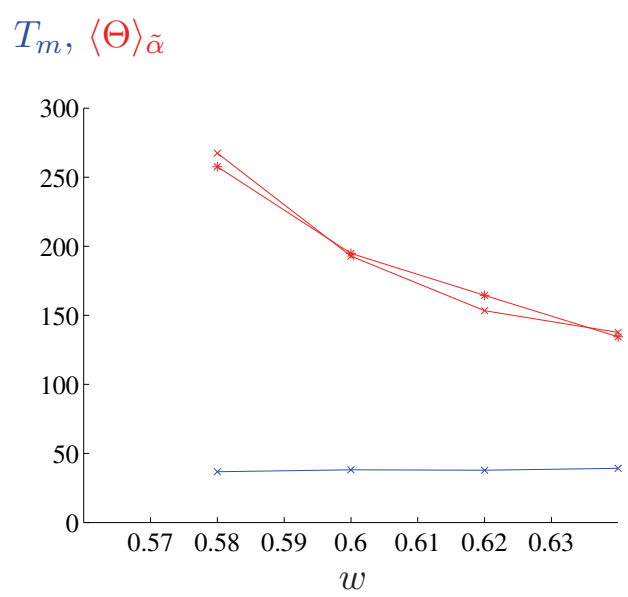

Figure 7. The period $T_{m}$ (lower curve in blue) of the macroscopic variable $m$ is compared to $\langle\Theta\rangle_{\tilde{\alpha}}$, the mean of all travel times of each of the two particle groups $\tilde{\alpha}$ (two upper curves in red). There is no simple integer ratio of the two and the ratio changes continuously through a range of values, so that one can assume that the oscillatory behavior results from a self-organizing effect and is not due to an influence from the periodic boundary conditions.

4. Numerical analysis using equation-free methods. In this section we investigate numerically in some detail the low-dimensional description of coarse scale features of the particle dynamics. This includes the rigorous exploration of parameter dependence on the macroscopic behavior, including the detection of bifurcation points, as well as the identification of its type and its two-parameter continuation. For this we apply and further develop methods of numerical bifurcation analysis, such that it is possible to obtain the necessary information in the framework of an equation-free analysis for a particle system with a medium number of particles, where fluctuations and discrete size effects cover a substantial part of the observed quantities. We are in particular interested in investigating what influence the value of the door width $w$ has on the macroscopic particle dynamics. Subsequently, we also investigate the influence of the relative velocity $r_{v^{0}}$.

For a first examination of the qualitative change of the macroscopic dynamic behavior and a later verification of our equation-free analysis, a brute force analysis is performed. In this, a forward and backward sweep (increasing and decreasing the door width $w$ ) is done, each sweep using the end state of the previous simulation as the new initial state when incrementing (respectively, decrementing) the door width $w$ by small steps $\Delta w$. For each simulation, we examine the asymptotic behavior numerically by plotting the extrema (minimum and maximum) of $m$ for the orbit of the final 30s of a 400s long simulation run, giving an indication of the size of the $\omega$-limit set.

Copyright (c) by SIAM. Unauthorized reproduction of this article is prohibited. 
Figure 8 shows both the forward sweep, from low to high $w$ (blue circles), and the backward sweep, from high to low $w$ (red crosses). For small values of $w$ the stationary "blocked" state is observed, while for values $w$ over a certain threshold, the system shows on the macroscopic level an oscillatory behavior. The growth of the amplitude as a function of the parameter $w$ is not inconsistent with the square root dependence on $w$ characteristic of a supercritical Hopf bifurcation.

For some system setups, with other parameters for the reaction time $\tau$ or other functions for the interaction potentials, we have observed in this type of backward and forward bruteforce sweep small separations between the stable stationary solution and the smallest value of the diameter of the stable limit cycle. Such separations could indicate that in a small region of parameter space, the actual bifurcation might be a subcritical Hopf bifurcation, signaling the presence of hysteresis. We concentrate here on system characteristics where this effect is negligible and the observed qualitative effects are in good agreement with a supercritical Hopf bifurcation.

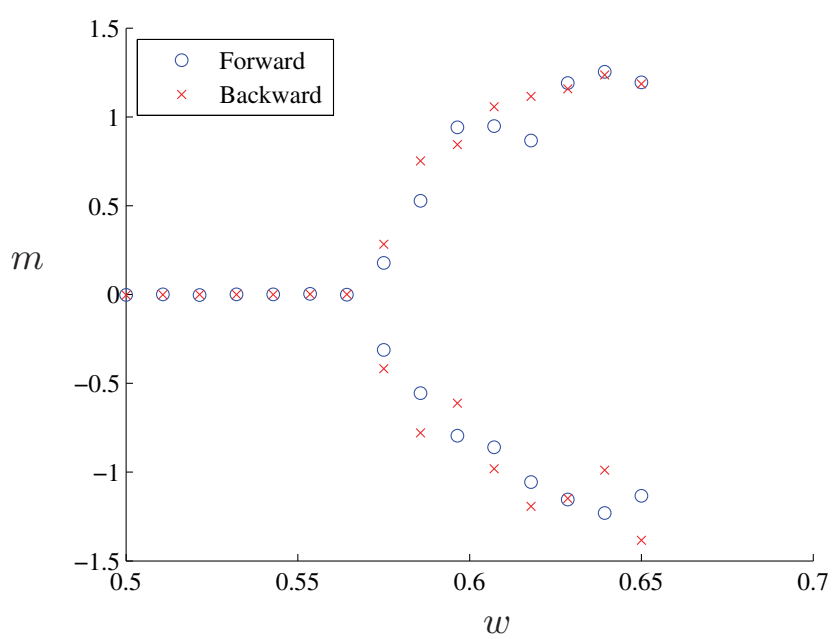

Figure 8. As an initial study, the qualitative change of the macroscopic dynamic behavior as a function of the door width $w$ is investigated by forward and backward sweeps, each using the end state of the previous simulation as the new initial state when incrementing (respectively, decrementing) $w$ by small steps $\Delta w=0.01$. For the limit cycle states, the values of the extrema (minimum and maximum) of the orbit of the last 30s of a 400s long simulation run are displayed. The plot shows that after the bifurcation the amplitude grows in a manner not inconsistent with the square root amplitude dependence characteristic of a supercritical Hopf bifurcation.

In the following, we further detect and identify the Hopf bifurcation point in the macroscopic variables $(m, \dot{m})$ by using the door width $w$ as parameter. After that, we perform an equation-free continuation of a fixed point including the detection of a Hopf bifurcation point and a subsequent two-parameter continuation of this Hopf bifurcation point using the relative velocity $r_{v^{0}}$ of the two species as the second parameter.

4.1. Numerical detection of the Hopf bifurcation point. In order to further examine the bifurcation we proceed to explore on a macroscopic scale the qualitative behavior around 
a stationary point first by a fit of a linearized equation of motion to the observed data and second by using Poincaré sections. The main difficulty here is for the equation-free approach to detect the necessary information, both noisy and influenced by finite size effects, from our microscopic particle simulation. This is also the reason why we have chosen to use two different approaches which allow us to cross-check the results.

To perform this analysis, the system is at each value of $w$ identically initialized by using a reference microscopic state, carefully chosen to be located close to the supposed stationary point. The microscopic initialization state is extracted from a reference simulation with a door small enough to force the system to oscillate down to the stable stationary point. See also section 4.2 for details about initializations of microscopic configurations. The macroscopic behavior with observables $(m, \dot{m})$ is then investigated for different values of the door width $w$ as the parameter.

Linearizing (7) around a stationary point, one will obtain a linear dynamical system in two dimensions of the form $\dot{x}=A x$, where we denote the eigenvalues of $A$ as $\lambda_{d}=a \pm i b$. The extraction of the real part of the eigenvalues is done by fitting the macroscopic time series $m(t)$ to the formal solution

$$
\varphi(t)=c_{1} e^{a t} \cos (b t)+c_{2} e^{a t} \sin (b t)+c_{3} .
$$

Since $c_{1}=\varphi(0)-c_{3}$, there are four parameters that must be estimated. A trust region method is used to perform the data fitting by employing the lsqnonlin command from MATLAB [34].

An estimator $\hat{T}$ for the period of the oscillation is found by Fourier analysis, giving an estimator of $\Im\left(\lambda_{d}\right)=\frac{2 \pi}{\hat{T}}$ that can be used as the initial guess in order to accelerate convergence of the parameter estimation. For values of $w$ smaller than the bifurcation value, where the stationary point is stable, the phase space trajectory will spiral into the stationary point and thus remain in the neighborhood where the linearization is valid. On the other side of the bifurcation, the trajectory is repelled away from the stationary point and thus away from the region where the linearization is valid. Care has therefore been taken only to fit the solution to the time series within a close neighborhood of the stationary point. A nonlinear least-square fit is then performed using a trust region method by employing the lsqnonlin command from the Optimization Toolbox in MATLAB [34] in order to estimate the four parameters, thus extracting the eigenvalues $a \pm i b$.

Because the simulations before the stability change do not feature oscillations permitting a clear estimation of the period of the signal, the constraint $b \in\left[\frac{2 \pi}{36}, \frac{2 \pi}{30}\right]$ is used on the imaginary part which we obtained by Fourier analysis. This technique permits an observation of a clear transition from negative to positive real parts of the eigenvalues $\lambda_{d}$ with increasing door width $w$. A summary of the results is displayed in Figure 9. This development of a numerical procedure being robust in detecting the stability information from the time series is necessary due to the fairly noisy nature of the microscopic model. The numerical investigation also shows that the imaginary part of the pair of complex conjugate eigenvalues is different from zero so that we can conclude that we indeed have a Hopf bifurcation.

To further verify the investigations of the change of stability in the presence of noise and discrete size effects, a second method is used, based on Poincaré sections. In order to obtain the maximum number of data points from a short trajectory, two half-lines are used as Poincaré sections. These are $m \geq 0$ and $m \leq 0$ at $\dot{m}=0$. Assuming that the linearization

Copyright (c) by SIAM. Unauthorized reproduction of this article is prohibited. 

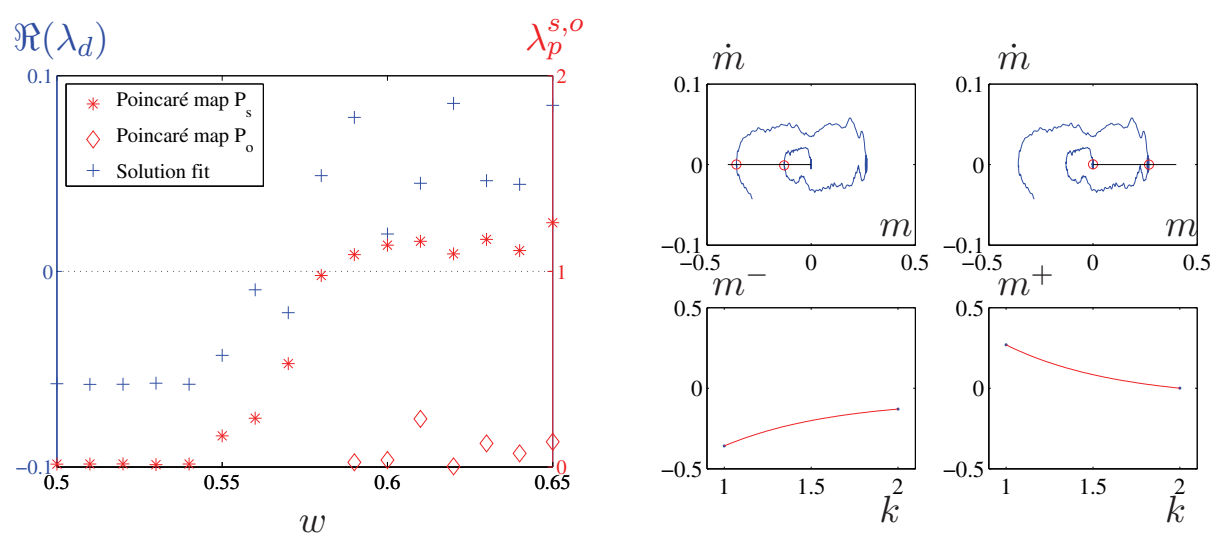

Figure 9. Numerical evidence of a Hopf bifurcation using two different methods. Left: As the bifurcation parameter $w$ increases, the real part $\Re\left(\lambda_{d}\right)$ of the eigenvalues $\lambda_{d}$ (of the linearization of the equation of motion (7) for the macroscopic state variables $m$ and $\dot{m}$ ) crosses from negative values to positive values. At the same time the imaginary part of the pair of conjugate complex eigenvalues is different from zero (not shown). The linearized equation of motion was obtained by fitting a solution to the differential equation to the data from the microscopic particle simulation. As an alternative method, Poincaré sections with eigenvalues $\lambda_{p}$ of linearized Poincaré maps are investigated. For the Poincaré map $P_{s}$, linearized at the stationary point, the change of the eigenvalue $\lambda_{p}^{s}$ with increasing $w$ from values smaller than one to values larger than one indicates a loss of stability of this stationary point. Furthermore, the linearized Poincaré map $P_{o}$, linearized at a point with $\dot{m}=0$ on the periodic orbit, is investigated. The values of $\lambda_{p}^{o}$ are smaller than one for values larger than those where the loss of stability of the stationary point is observed, indicating the existence of a stable periodic orbit. Right: The Poincaré sections ( $m \leq 0$ and $m \geq 0$ ) in phase space and the solutions interpolated between the discrete values of $k$ are shown for $w=0.55$. These solutions are used subsequently to estimate the eigenvalues $\lambda_{p}^{s}$ of the linearized Poincaré map $P_{s}$.

is identical for both half-lines, the (eigen)value $\lambda_{p}$ of the linearized Poincaré map of the type $m_{k+1}=\lambda_{p} m_{k}$ is the same for both maps $P_{s}^{+}$and $P_{s}^{-}$around the stationary point. By fitting each sequence $m_{k}^{+}$and $m_{k}^{-}$to the solutions

$$
\begin{aligned}
& m_{k}^{+}=\left(\lambda_{p}\right)^{k} m_{0}^{+}, \\
& m_{k}^{-}=\left(\lambda_{p}\right)^{k} m_{0}^{-},
\end{aligned}
$$

the common eigenvalue can be determined. To distinguish between Poincaré maps near the stationary point and near the orbit, we use $\lambda_{p}^{s}$ and $\lambda_{p}^{o}$, respectively.

To obtain stability information of the fixed point under investigation, points $\left(m_{k}^{ \pm}, 0\right)$ on the limit cycle are excluded when estimating the eigenvalue $\lambda_{p}$ of the Poincaré map. The plot in Figure 9 shows a transition from a stable to an unstable stationary point at a critical value of the door width $w_{0}$, close to the one obtained with the approach based on the linearization of the differential equation and fitting the solution to data. For parameters larger than $w_{0}$, additional Poincaré sections (additional to the Poincaré sections in the neighborhood of the stationary state) show the presence of a stable periodic orbit. The corresponding Poincare section is called $P_{o}$. Linearizing $P_{o}$ around the intersection point $\bar{m}^{o}$ of the orbit with the line $\dot{m}=0$ results in $m_{k+1}^{o}-\bar{m}_{0}^{o}=\lambda_{p}^{o}\left(m_{k}^{o}-\bar{m}_{0}^{o}\right)$ with the solution

$$
\left(m_{k}^{o}-\bar{m}^{o}\right)=\left(\lambda_{p}^{o}\right)^{k}\left(m_{0}^{o}-\bar{m}^{o}\right) .
$$

Copyright $\odot$ by SIAM. Unauthorized reproduction of this article is prohibited. 
Taken together, the transition from a stable stationary point to an unstable one, surrounded by a stable limit cycle with monotonically increasing amplitude for values larger than $w_{0}$, is strongly indicative of a supercritical Hopf bifurcation.

4.2. Equation-free continuation and lifting near a stationary point. To supplement and complete the information about the macroscopic system behavior and its dependence on a parameter, the aim is to perform a numerical bifurcation analysis for the macroscopic properties. Since no explicitly given equations are available for a description of the chosen macroscopic variables $m$ and $\dot{m}$, we apply an equation-free approach [26], in which the necessary information is obtained by carefully selected short simulation bursts of the underlying microscopic particle model. For the continuation of a stationary point of the macroscopic dynamics of (7) we use a predictor-corrector approach (see, for example, [7], [8], [4]), but in contrast to standard applications, the right-hand side $F$ is unknown in the considered situation.

Information about $F$ can be obtained by switching between the macroscopic level, where no equations are available, and the microscopic level, where our model is given [26]. A typical choice for the predictor would be a constant or a linear predictor, and a Newton method for the corrector. For the investigation of stable objects in phase space it is even possible to replace the Newton method with a simple direct simulation of the microscopic model. The essential information from the right-hand side of $(7)$, like the Jacobian $\frac{\partial F}{\partial x}$ with $x=(m, \dot{m})$ for the Newton method as the corrector, is obtained by numerical evaluation of the microscopic model. To obtain this information at predicted points in a neighborhood of the stationary point, it is necessary to switch between the macroscopic level and the microscopic level [26]. The change from the microscopic level to the macroscopic level is called restriction $\mathcal{R}$, and the change from the macroscopic description to the microscopic particle model is called lifting $\mathcal{L}$; see Figure 10 for illustrations. In systems like the present, where the available numerical information for computing a Jacobian is very noisy, it is favorable to use another corrector. In the present paper we use the false position method [40] to numerically determine zeros of the right-hand side of (7). The false position method is more robust and can even deal with functions with discontinuities. To be able to also continue along fold bifurcation points, a pseudoarclength predictor-corrector method can be used [7], [8], [4].

The restriction $\mathcal{R}$ is the unique map $S \mapsto(m, \dot{m})$ taking a microscopic state $S$ to a macroscopic state $(m, \dot{m})$ by means of the defining equation (6) for $m$ and its time derivative $\dot{m}$. The lifting $\mathcal{L}$ is not unique, as it describes a map from a small number of macroscopic variables to a microscopic model with many degrees of freedom. Therefore, the applicability of this approach requires the system property that the system converges quickly to a low-dimensional behavior which is typically satisfied for all systems showing pattern formation. This includes the need to lift to a "physically meaningful" or "natural" state which is in a close neighborhood of the macroscopic state under investigation. The neighborhood should be such that the process of converging back to the low-dimensional manifold occurs on a time scale much smaller than the dynamical processes on the macroscopic level. Microscopic configurations far away from this low-dimensional manifold would not represent the macroscopic behavior of interest. Detailed knowledge about the application problem considered has to be used to be able to construct a good and effective lifting operator.

Copyright (c) by SIAM. Unauthorized reproduction of this article is prohibited. 


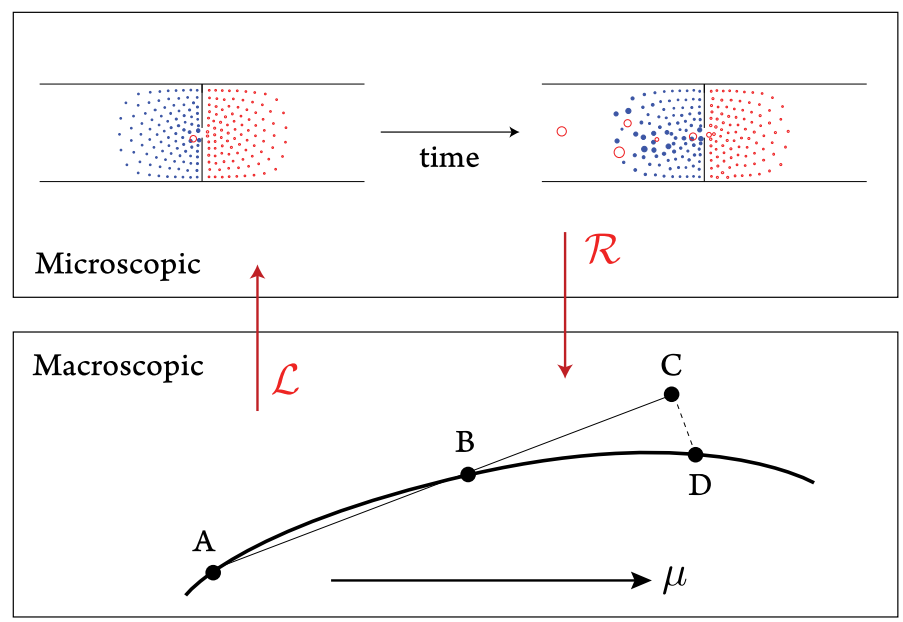

Figure 10. Sketch of the equation-free approach. Information from microscopic simulations is transferred to the macroscopic description utilized for a continuation procedure. For the macroscopic description we compute the macroscopic variables $(m, \dot{m})$ from the microscopic information (restriction $\mathcal{R})$. In the reverse procedure $($ lifting $\mathcal{L})$, a microscopic state corresponding to a given value $(m, \dot{m})$ is computed. For the systems under consideration, many possible microscopic configurations exist for a given macroscopic state, but those different microscopic configurations all typically converge quickly to the same macroscopic behavior. This behavior is checked in Figure 13. The lower part of the figure sketches the numerical continuation procedure of a stationary state depending on the parameter $\mu$ employing a pseudoarclength predictor-corrector method: A secant prediction using the points $A$ and $B$ results in the predicted point $C$. With $C$ as the initialization for the corrector, the point $D$ is obtained.

4.2.1. Construction of a lifting operator. To be able to perform an equation-free continuation of the macroscopic variables, we require a lifting operator $\mathcal{L}$ which constructs, for the chosen particle model, a microscopic system configuration close to the point under investigation on the low-dimensional manifold in state space. Being close to this low-dimensional manifold, well characterized by the macroscopic variables $(m, \dot{m})$, results in a rapid convergence back to this manifold. This ensures then that in very good approximation, the analysis operates on the low-dimensional manifold of interest.

Obtaining a microscopic configuration close to a stationary point can be done by letting the system settle to the $\omega$-limit set of a stable stationary state. In order to prepare for the lifting, a collection of numerical experiments with steady state situations is computed for values of $r_{v^{0}}>1$. It is assumed that the unstable stationary states are microscopically similar to the stable stationary states, and thus the same lifting $\mathcal{L}$ is used.

By investigating the particle distribution of the microscopic structure of the $\omega$-limit set, it is observed that to a very good approximation, the number of particles positioned in small bins of size $\Delta x=0.2$ depends linearly on the particles' distance to the door. This is shown in Figure 11. The slope and intercept describing the linear distribution are linearly dependent on the relative velocity $r_{v^{0}}$. Furthermore, it has been checked that those parameters are independent of the door width $w$.

To construct the microscopic configuration of a stationary state for the lifting operator $\mathcal{L}$, particles are placed according to the distribution found. As explained and shown in Figure

Copyright (c) by SIAM. Unauthorized reproduction of this article is prohibited. 

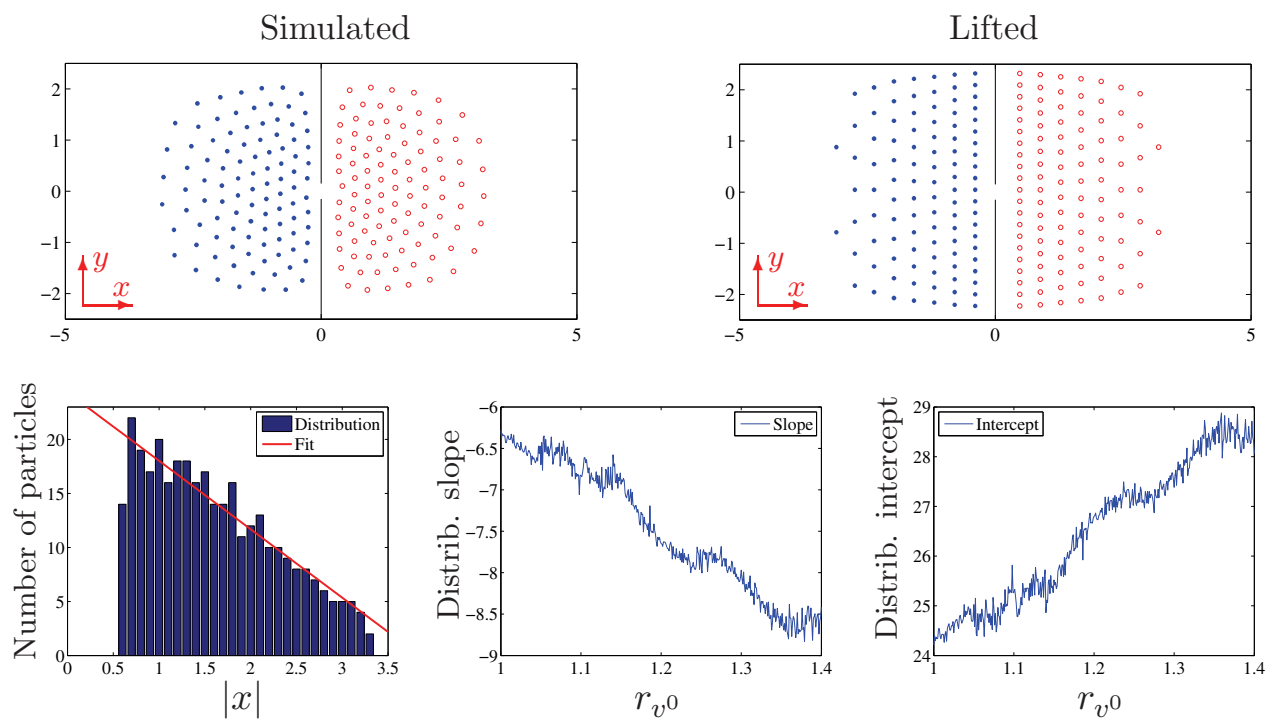

Figure 11. In states near the equilibrium, the number of particles at distance $|x|$ from the door decreases linearly with $|x|$. For this linear function (the best line fit to the distribution of particle numbers within bins of size $\Delta x$, bottom left) the slope and the intercept (bottom center and right) are found to depend nearly monotonically on the parameter $r_{v^{0}}$. In the lifting procedure, a microscopic configuration of a stationary state is then constructed by placing particles according to such a distribution in the $x$-direction and equidistantly distributed in the $y$-direction.

11 , the slope and intercept of the distribution depend on the relative velocity $r_{v^{0}}$ but are independent from the door width $w$. In this construction, particles are equidistantly distributed in each layer. The distance between the vertical layers is chosen to have the value 0.39 , which minimizes a microscopic distance measure $\gamma$ between a simulated reference state $S_{1}$ and this lifted microscopic state $S_{2}$ (see Figure 11). Setting the velocities of all particles to zero ensures $\dot{m}=0$.

Initializing a nonstationary state located in the neighborhood of a stationary state is achieved by creating a small lane of particles having just passed the door. For a lane of $l$ particles, the distributions are used to construct two crowds with $N / 2-l$ and $N / 2+l$ particles, where $N$ is the total number of particles. The microscopic state with the lane is then constructed by converting $l$ particles on the relevant side to the opposite species. The particles to be converted are selected as the particles in the $l$ first layers in the center of the corridor. This lifting is by nature restricted to discrete values of $m$ obtained by constructing all possible lane lengths on each side. By giving all particles zero velocity, we restrict ourselves to lifting to states having $\dot{m}=0$. This simple choice of the lifting procedure is sufficient because our object of interest is a closed curve in phase space which we investigate closer by Poincaré sections, following the macroscopic dynamics for a full period; the result is independent of the starting point on the curve.

The microscopic distance measure used in the construction of the lifting procedure is

$$
\gamma\left(S_{1}, S_{2}\right)=\left\|\mathbf{d}_{1}-\mathbf{d}_{2}\right\|
$$

Copyright (c) by SIAM. Unauthorized reproduction of this article is prohibited. 
where the Euclidean norm is used for the difference between two discretized density profiles $\mathbf{d}_{1}$ and $\mathbf{d}_{2}$, each profile being a vector of densities evaluated at discrete positions from a grid size of $0.2 \times 0.2 \mathrm{~m}^{2}$ over the corridor. See Figure 12 for a density profile. In order to obtain a smooth density profile, the density $d(x, y)$ at a specific grid point is computed by summing up the contribution of each particle $i$, weighted by the $\kappa(\cdot)$ function introduced in (5). This results in

$$
d(x, y)=\sum_{i} \kappa\left(x_{i}\right) K\left(\sqrt{\left(x_{i}-x\right)^{2}+\left(y_{i}-y\right)^{2}}\right),
$$

where the function $K(\cdot)$ weights points higher the closer they are to the grid point. $K(\cdot)$ is chosen as the Epanechnikov kernel function [10] shown in Figure 12

$$
K(u)= \begin{cases}\left(1-\left(\frac{u}{h}\right)^{2}\right), & \left|\frac{u}{h}\right|<1, \\ 0, & \left|\frac{u}{h}\right| \geq 1 .\end{cases}
$$

The steepness of $K(u)$ is controlled by the bandwidth parameter $h$.
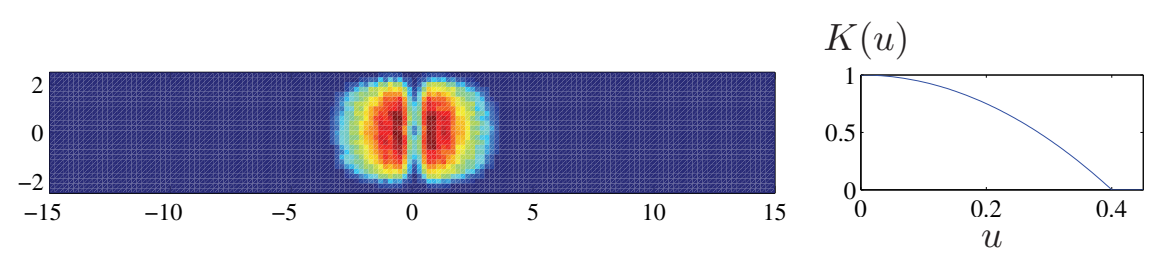

Figure 12. Two-dimensional density profile of a stationary state computed with a bandwidth $h=0.4$ and a grid size 0.2 . The densities range from 0 to 13.4 particles $/ \mathrm{m}^{2}$. The kernel density function $K(u)$ is used to obtain smooth estimates of the density of the particles.

As mentioned earlier, the usability of a lifting operator $\mathcal{L}$ depends on how close the state of the microscopic system can be initialized to the low-dimensional manifold determining the observed macroscopic behavior in the high-dimensional state space. If the lifted microscopic state converges quickly to the low-dimensional manifold of interest, one can discard without a big numerical error the first part of the trajectory before the relevant macroscopic behavior is observed. Therefore we test this rapid convergence behavior for the states obtained by the introduced lifting operator $\mathcal{L}$. For this, the $\gamma$ measure defined in $(8)$ is used to assess how quickly a lifted stationary state $L_{0}$ converges towards the microscopic reference stationary state $S_{0}$ by examining how quickly the quantity $\gamma\left(L_{0}(t), S_{0}\right)$ decreases to the noise level compared to the other variables in phase space (Figure 13). Recall that by construction, the macroscopic variable $m$ is the mean of the density profile in the $x$-direction. Consequently, computing $m$ can be seen as a projection of the density profile obtained from a microscopic state onto a point $m$, yielding a significant reduction of the number of variables. In addition to the lifted states we also tested the rapid convergence of perturbed microscopic states in general. In order to assess whether the $(m, \dot{m})$ variable is representative for the microscopic system, this microscopic state is perturbed by a high-dimensional random vector with mean 0 and variance 0.1 . The rapid convergence of the perturbed microscopic states to the lowdimensional manifold in Figure 13 provides good justification for the choice of the macroscopic variables and the equation-free approach. Furthermore, it can be observed that there is no

Copyright $\odot$ by SIAM. Unauthorized reproduction of this article is prohibited. 

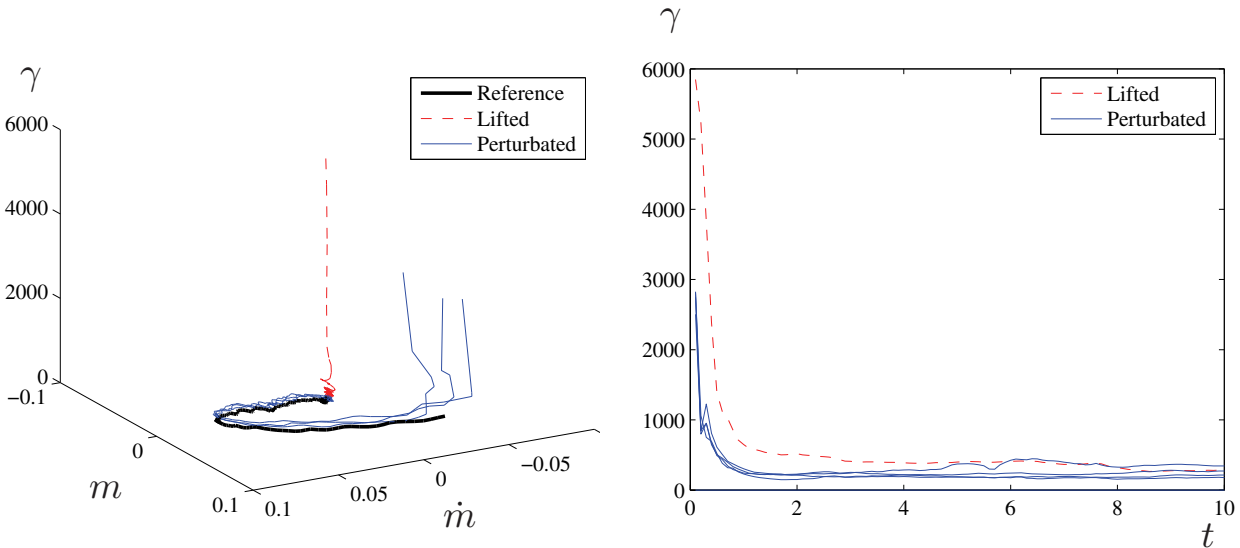

Figure 13. The lifting $\mathcal{L}$ is evaluated by measuring the distance $\gamma$ of the resulting microscopic state $L$ to the microscopic reference state. Left: One reference simulation, compared to four others having their initial conditions perturbed by randomly adjusting velocities and positions of each particle. Compared to the other time scales in the system, a rapid convergence to the reference state is observed. The evolution from the lifted state to the low-dimensional manifold is depicted as a dashed red line. Observe that there is no relevant change of $(m, \dot{m})$ in the process of convergence. This is a further demonstration of the validity of the approach developed here. Right: Plot of the distance $\gamma$ over time showing the convergence of the perturbed simulations to the low-dimensional manifold.

relevant change of $(m, \dot{m})$ in the convergence from the lifted state to the low-dimensional manifold. This is a further demonstration of the validity of the approach developed here.

4.2.2. Equation-free investigation of the stationary state. To be able to investigate the parameter-dependent qualitative changes of the macroscopic behavior of the considered particle model, we first perform an equation-free continuation of the stationary state of (7),

$$
F\left(m, \dot{m} ; w, r_{v^{0}}\right)=(0,0),
$$

with the macroscopic variable center of mass $m$, its derivative $\dot{m}$, and parameters door width $w$ and relative velocity $r_{v^{0}}$ defined in (4). As (7) is not explicitly known and the numerical information about $F$ obtained from the described equation-free approach is rather noisy, we consider a state to be stationary if (9) is satisfied within a certain tolerance. Stationary states in that respect are considered to be stable if they fulfill an adapted Lyapunov stability criterion for a neighborhood $U$ of fixed size defined by the noise level. This means that a stationary state in the above sense is called stable if solution curves initialized in $U$ remain in $U$ for all (computed) times. It should be remarked that the expressions about stationarity and stability are used in the described sense also in the following without mentioning it each time.

As the right-hand side $F$ expresses the time derivative of the vector $(m, \dot{m})$ resulting in the vector $(\dot{m}, \ddot{m})$, evaluation of $F$ amounts to a differentiation of our macroscopic variable $m$. As we explained after (6), we use central derivatives to obtain a more robust computation of the derivative. Using the parameter $\Delta t=0.1$ we observed for the numerically computed $\dot{m}$ a very smooth looking curve. The computation of the second time derivative to obtain $\ddot{m}$ was also done with central derivatives and resulted, as expected, in a much noisier time series.

Copyright ( $\odot$ by SIAM. Unauthorized reproduction of this article is prohibited. 


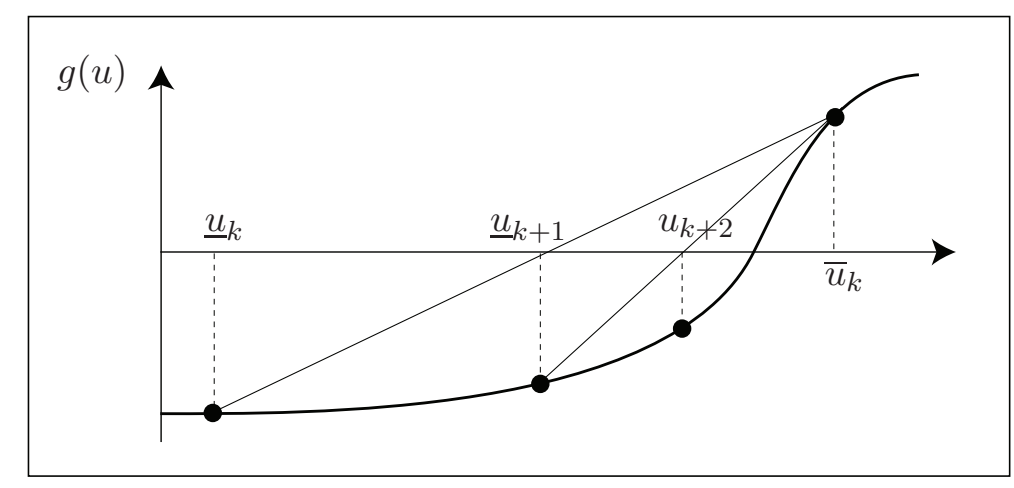

Figure 14. Sketch of the false position method to determine the zero of a function $g(u)$. Each iteration is obtained by connecting a line between a negative value point $\left(\underline{u}_{k}, g\left(\underline{u}_{k}\right)\right)$ and a positive value point $\left(\bar{u}_{k}, g\left(\bar{u}_{k}\right)\right)$, where $g\left(\underline{u}_{k}\right)<0$ and $g\left(\bar{u}_{k}\right)>0$. The zero of this line is then used as one of the points in the next iteration, together with the point of the previous iteration that has the opposite sign. This iteration is continued until $\left\|\underline{u}_{k+1}-\bar{u}_{k+1}\right\|$ is smaller than a predefined tolerance $u_{\mathrm{tol}}$.

For the results presented in the following, even for this noisy second derivative obtained by $\Delta t=0.1$ our suggested algorithms gave consistent results, but we also checked other parameters up to $\Delta t=1.5$ naturally resulting in much smoother curves (since this effectively averages over some time interval). These, however, gave rise to the same bifurcation diagrams as those being presented in the following.

The equation-free continuation of the stationary point is based on a predictor-corrector method with a constant predictor and a false position method as the corrector. A constant predictor is used when incrementing the door width, meaning that the microscopic state for the previous door width is used as the initialization for the next one. In order to correct, we need to find, in a very robust manner (because of the noise in the system), the zero of a function $g(\cdot)$, in this case being $g(w)=F_{2}=\ddot{m}$, as $F_{1}=\dot{m}$ is zero by construction of the lifting. To this end we use the false position method [40]. This method is extremely robust and assumes very few properties of the function $g$. For the following we assume a single sign change of $g$. Starting with two points $\underline{u}_{0}$ and $\bar{u}_{0}$ such that $g\left(\underline{u}_{0}\right)<0$ and $g\left(\bar{u}_{0}\right)>0$, the false position method (see Figure 14) proceeds by producing a nested sequence of intervals $\left[\underline{u}_{k}, \bar{u}_{k}\right]$ that all contain a sign change of $g$, i.e., $g\left(\underline{u}_{k}\right)<0, g\left(\bar{u}_{k}\right)>0$ and $\left\|\underline{u}_{k+1}-\bar{u}_{k+1}\right\|<\left\|\underline{u}_{k}-\bar{u}_{k}\right\|$ for all $k$. The method is terminated when the interval size reaches a certain tolerance value $u_{\text {tol }}$ (in this case $u_{\text {tol }}=0.05$ is chosen) or if the lifting resolution is insufficient to shrink the interval further. At iteration number $k$, the value

$$
u_{k+1}=\frac{g\left(\bar{u}_{k}\right) \underline{u}_{k}-g\left(\underline{u}_{k}\right) \bar{u}_{k}}{g\left(\bar{u}_{k}\right)-g\left(\underline{u}_{k}\right)}
$$

is computed, being the root of the line through $\left(\underline{u}_{k}, g\left(\underline{u}_{k}\right)\right)$ and $\left(\bar{u}_{k}, g\left(\bar{u}_{k}\right)\right)$. If $g\left(\underline{u}_{k}\right)$ and $g\left(u_{k+1}\right)$ are equal, then $\underline{u}_{k+1}$ is set to $u_{k+1}$ and $\bar{u}_{k+1}$ to $\bar{u}_{k}$; otherwise, $\underline{u}_{k+1}$ is set to $\underline{u}_{k}$ and $\bar{u}_{k+1}$ to $u_{k+1}$. Iterating, the sequence $u_{k}$ will approach the zero or the sign change of $g(u)$. To obtain $g(m)=\ddot{m}, F$ is measured 0.4 s after the initialization, in order to ensure convergence to the low-dimensional manifold while staying as close as possible to the initialization point.

Copyright $\odot$ by SIAM. Unauthorized reproduction of this article is prohibited. 
Starting the continuation at door width $w=0.4$ with a step size of $\Delta w=0.02$, we observe that the stationary point is located in a neighborhood of zero defined by the noise level which does not vary with $w$; see Figure 15 .

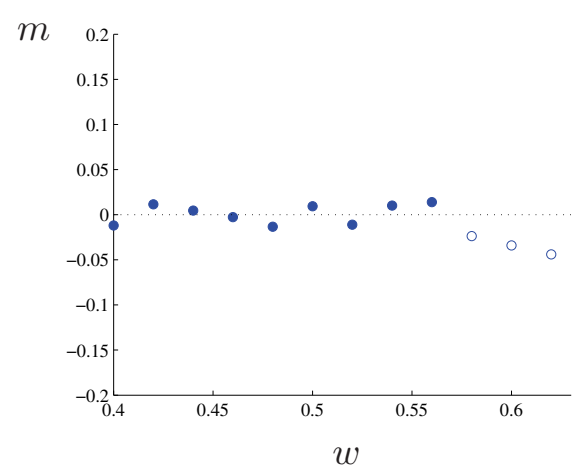

Figure 15. Continuation of the stationary point at $r_{v^{0}}=1$ with a constant predictor, using a false position method as the corrector. The lifting $\mathcal{L}$ was done as described in section 4.2.1. The filled circles correspond to stable stationary points and the open circles to unstable stationary points.

Stationarity is investigated for each door width $w$ by checking whether (9) is satisfied within a certain tolerance. Because of the lifting to states with $\dot{m}=0$, it is sufficient to investigate $g(m)=\ddot{m}$, measured 0.4 s after the microscopic initialization in order to ensure convergence to the low-dimensional manifold. The tolerance used is 0.05 .

Along the continuation of the stationary point, stability is examined by the adapted Lyapunov stability criterion described above. If the system state remains in the neighborhood $U$ of the investigated stationary point, this point is considered stable in the above sense. The adapted Lyapunov stability is checked during a 50s long simulation. If the system state remains, at all times, in the region $U$ defined by the distance 0.05 for $m$ and 0.1 for $\dot{m}$ to the stationary point of the macroscopic dynamics (7), then the system is considered stable.

If the above values are inappropriately chosen, wrong results could be obtained. Therefore, our findings were verified by the examination of a representative selection of simulations. The tolerance ranges were chosen finding estimates for the noise level from a number of simulations.

Combining the continuation of the stationary point together with the findings in section 4.1 gives us a Hopf point for $r_{v^{0}}=1$ which is used in the following section for the two-parameter continuation.

4.3. Two-parameter continuation of the Hopf point. Having found a bifurcation point, here a Hopf point, by continuation of a stationary state with respect to one parameter, a natural question is how the location of this bifurcation depends on a second parameter. The parameters used for the investigation of the qualitative changes of the macroscopic behavior are the door width $w$ and the relative velocity $r_{v^{0}}$. As a result, one obtains regions in this two-parameter plane with solutions of the same qualitative behavior. Here, a region of stable fixed points is separated by a line of Hopf points from a region characterized by oscillatory behaviors. In the equation-free framework, a two-parameter continuation is difficult, as all the available macroscopic system information typically is very noisy. This has been made

Copyright (c) by SIAM. Unauthorized reproduction of this article is prohibited. 
possible only by the development and combination of robust numerical algorithms tailored to the equation-free analysis.

Starting with the Hopf point, found by the fixed point continuation for $r_{v^{0}}=1$ described in section 4.2.2, a two-parameter pseudoarclength continuation is then performed. Following the procedure of a pseudoarclength continuation we apply a linear predictor using the secant of the two last points and subsequently a false position method as the corrector, where we search in a direction perpendicular to the predicted direction. This results in a line search in a subspace of the two-dimensional plane $\left(w, r_{v^{0}}\right)$ (Figure 16), locating the change of stability of the stationary state. This line, having the direction vector $\mathbf{v}$, can be parametrized as $u \mathbf{v}$, with parameter $u \in \mathbb{R}$.

As explained in section 4.2.1, the lifted state is by construction located in a small neighborhood of the stationary point. This ensures that $F$ in (7) remains small, thus being in good approximation of the stationarity condition, so that the line search can be reduced to the detection of a stability change.

Because all derivative information is unknown or very noisy, the previously used false position method [40] is also applied here to find the stability change by searching for a sign change of a specifically constructed stability function $g$. This function $g$ is defined as a sign function indicating stability by the previously described adapted Lyapunov stability criterion (see section 4.2.2). When the system is stable, the value -1 is assigned to the function $g$ (marked with blue in Figure 16), and 1 otherwise (marked in red). During a line search, a Hopf point is detected if a sign change of $g$ is detected between two points of distance less than 0.05 in the $\left(w, r_{v^{0}}\right)$-plane. This restriction to binary values is done to make the procedure more robust.

We expect this line, obtained by the equation-free continuation, to represent Hopf bifurcation points. In principle, we cannot rule out that bifurcations of higher co-dimension occur along this line, but the determined stability change ensures that we are always following a bifurcation point.

To verify the numerical results obtained by the equation-free approach, direct simulations are used for three values of $r_{v^{0}}$, as seen in Figure 16(a). Furthermore, the macroscopic variable $m$ is plotted against $w$ for the stationary states of those direct simulations (Figure 16(b)). Each point is measured $0.4 \mathrm{~s}$ after the initialization in order to ensure convergence to the low-dimensional manifold.

The comparison of the period $T_{m}$ of the macroscopic variable $m$ to $\langle\Theta\rangle_{\tilde{\alpha}}$, the mean of all travel times of each of the two particle groups $\tilde{\alpha}$, is also made for values $r_{v^{0}}>1$. Here, $\tilde{\alpha}$ represents those particles of one species that completed a travel cycle in the observation time. This travel time is measured only for cases where a stable orbit is present. The comparison is shown in Figure 17. As result, the argument from section 3 and Figure 7 is extended to other values of the relative velocity $r_{v^{0}}$ : There is no simple integer ratio of the two times, so it can be assumed that the oscillatory behavior results from a self-organizing effect and is not due to an influence from the periodic boundary conditions.

For larger relative velocities $r_{v^{0}}$, the initial change of position of the bifurcation point to smaller values of the door width $w$ than for $r_{v^{0}}=1$ is due to the simple reason that the more energetic particles are able to push through and initiate oscillations at a door width smaller than when the two populations were of equal average energy.

Copyright $\odot$ by SIAM. Unauthorized reproduction of this article is prohibited. 
(a)

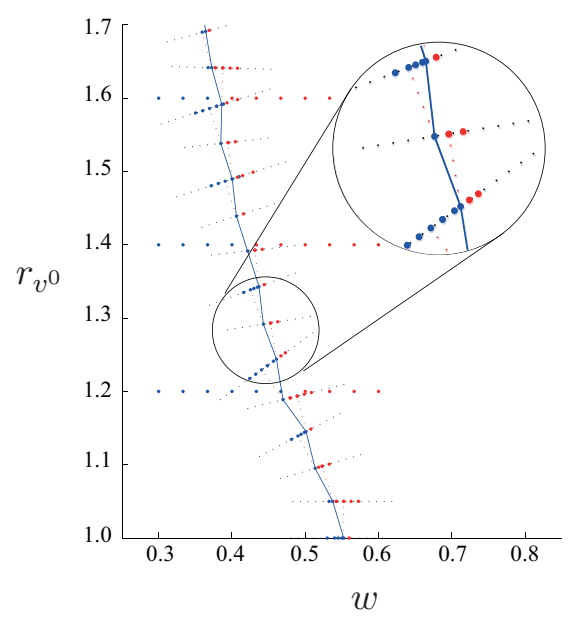

(b)

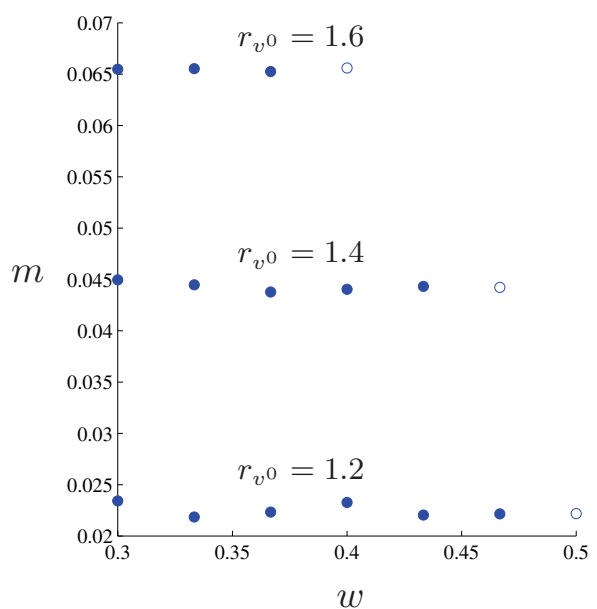

Figure 16. (a) Bifurcation diagram obtained by an equation-free two-parameter continuation of a Hopf bifurcation point. The curve of Hopf bifurcation points separates regions of two different qualitative behaviors in the macroscopic properties of the particle system. The thin dashed lines (see also the magnified area) show the search directions for the false position method as the corrector. This direction is chosen perpendicular to the predicted direction, in order to obtain a pseudoarclength method. The points marked are the predicted ones and those used as intermediate points for the corrector which were checked for their stability properties and colored accordingly. As a result one obtains on the left side of the line of Hopf bifurcation points stable fixed points (in blue) and on the right unstable fixed points (in red). On the right of this separating line of Hopf points we have an oscillatory region where the existence of stable limit cycles was tested by Poincaré sections as described in section 4.1. The three horizontal dotted lines show stability results of direct simulations to verify the equation-free continuation. (b) For the three values of $r_{v}$ tested with the direct simulations of (a), the macroscopic variable $m$ is plotted against $w$ for the stationary states. Filled circles represent stable states and open circles unstable states.

As the velocity ratio $r_{v^{0}}$ increases further, the periodic orbit shifts in $m$, resulting in periodic bursts of one species only for values of $w$ close to the bifurcation point. The different onset of oscillations for each species periodically passing the door is seen in Figure 17.

5. Conclusions and outlook. The methods introduced in the present paper have permitted us to perform equation-free numerical bifurcation analysis for a particle model and could help the future development of more rigorous methods to optimize building geometry for better crowd control.

We have described a class of particle systems with an intermediate number of particles. The particles interact mutually and with the geometry of the surroundings. We focus on a situation where two species of particles compete for passage through a doorway, aiming in opposite directions. We have studied the time evolution of macroscopic, or coarse-grained, variables $(m, \dot{m})$ as the system undergoes a transition from the doorway being effectively blocking, to the doorway being large enough to permit an oscillating flux, and eventually a transition to a nearly free-flow regime. The aim has been to examine and to develop numerically robust methods for this, as well as to model pedestrian crowds. This work extends previous modeling efforts (see, e.g., [21]) by employing more realistic finite range interactions 

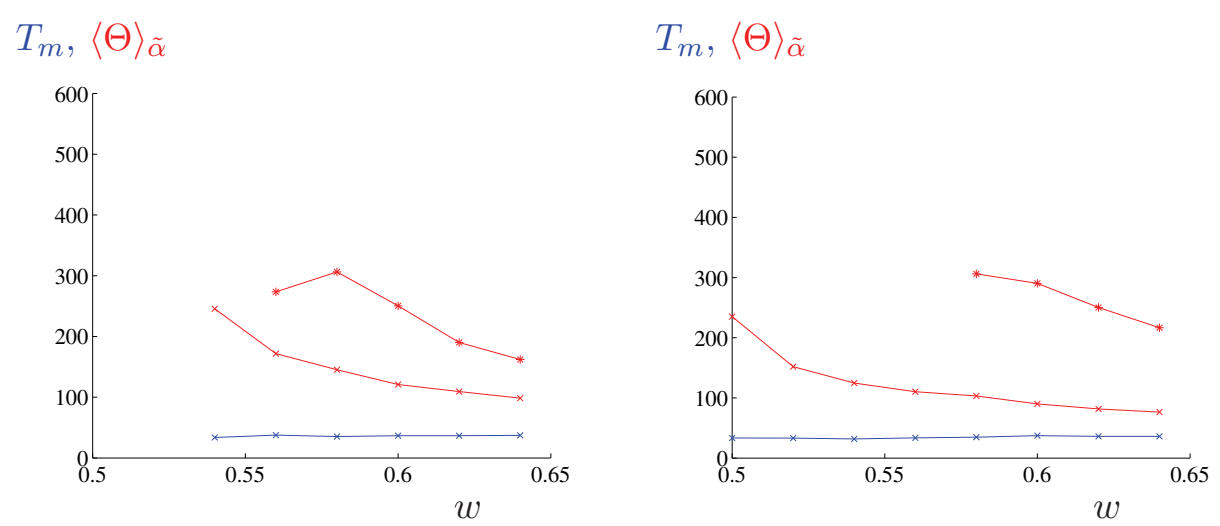

Figure 17. As in Figure 7, the period $T_{m}$ (lower curve in blue) of the macroscopic variable $m$ is compared to $\langle\Theta\rangle_{\tilde{\alpha}}$, the mean of all travel times of each of the two particle groups $\tilde{\alpha}$ (two upper curves in red). The two graphs show for different relative velocities $r_{v 0}$ that there is no simple integer ratio of the two and the ratio changes continuously through a range of values, so that one can assume that the oscillatory behavior results from a self-organizing effect and is not due to an influence from the periodic boundary conditions. The curves start after the Hopf bifurcation point or after the respective crowd starts traveling. Left: $r_{v^{0}}=1.1$. Right: relative velocity $r_{v^{0}}=1.2$. The difference in the onset of the two red lines illustrates a region in which only species of one kind are periodically bursting through.

among the particles and adds an equation-free numerical analysis of these systems to the literature.

The principal findings are the following: (1) We can reproduce and quantify previous results on simulations of pedestrian behavior. (2) We can classify regions in a two-dimensional phase space of macroscopic variables and characterize the transition between them as a supercritical Hopf bifurcation. (3) We can continue the Hopf bifurcation point in a two-parameter space for the door width and the relative velocity of the two pedestrian species as parameters. (4) In addition to this, we demonstrate a two-parameter continuation in an equation-free setting which gives rise to a number of technical challenges. To achieve this, we employ a novel combination of a number of robust numerical algorithms.

The nontrivial challenges which were encountered are the following: By their nature, finite size particle systems behave noisily. As a consequence, the equation-free procedure, going back and forth between high-dimensional and low-dimensional descriptions, is difficult, and the extraction of meaningful information requires careful and robust numerical algorithms. In addition to this, the finite size models have boundary conditions which may interfere with the observed macroscopic phenomena, and care must be taken to avoid or control this, in particular when we make one species more agile, i.e., change the relative velocity of the two pedestrian species.

The particle models we have investigated for describing pedestrian flow through a narrow doorway have similarities with fluid dynamics phenomena reported in [28], [29] of oscillations in the flow of water forced vertically by gravity through a vertical bottleneck. The analogy arises if one crowd is interpreted as the water in the bottle and the other crowd as the inflowing air. Consistent with the phenomena we observe in the present paper, these investigators found that by gradually increasing the diameter of the opening for an upside-down bottle, states of 
no flow, oscillatory flow, and counterflow were observed. Observations of the fluid behavior for more complicated situations like the shape and geometry of the area which is met by the outflowing fluid [37], as well as the coupling of several fluid systems of this type [30], may in turn inspire future work for pedestrian flows.

For discrete particle models, an obvious goal for further investigations is to understand and possibly optimize emergency exits and other crowd constraining constructs, like subway stations or stadium entries and exits. This important topic is also addressed in [18].

Quantitative experiments with actual pedestrians are not abundant in the literature (see, however, the recent work [36] and the references therein), but nevertheless they are very much needed in order to tune and validate the interacting particle model of crowds. In particular, the onset of lane formation for certain densities and certain ratios of crowd agility represents both an important qualitative change in the pedestrian flow and a challenge to further modeling.

For very high values of $r_{v^{0}}$, situations where there is a steady flow of species through the door, the possibility of another bifurcation changing from the oscillatory to a steady state is opened.

Even further opening of the doorway, exceeding the range of the door width investigated in the present paper, makes the macroscopic behaviors approach those corresponding to no doorway. A typical macroscopic pattern is seen in Figure 18. The two counterstreaming populations segregate into "lanes" containing a few particles in the cross-corridor direction and many particles along the corridor. To be able to distinguish situations with multiple lanes from a single lane requires additional macroscopic variables, which implies further challenges for the numerical analysis. The analysis of this regime could be of interest for further research.

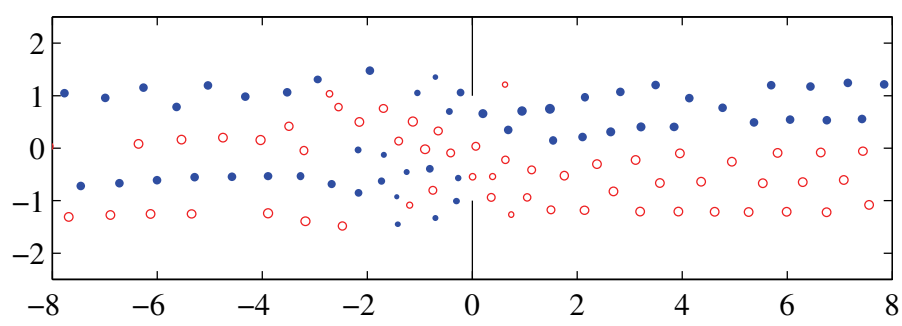

Figure 18. Structural change at very large door widths, showing formation of lanes. The radii of the circles are proportional to pedestrian velocity.

\section{REFERENCES}

[1] G. Antonini, M. Bierlaire, And M. Weber, Discrete choice models of pedestrian walking behavior, Transportation Res. Part B, 40 (2005), pp. 667-687.

[2] N. Bellomo And C. DogBe, On the modeling of traffic and crowds: A survey of models, speculations, and perspectives, SIAM Rev., 53 (2011), pp. 409-463.

[3] A. J. Bernoff And C. M. Topaz, A primer of swarm equilibria, SIAM J. Appl. Dyn. Syst., 10 (2011), pp. 212-250.

[4] W. J. Beyn, A. Champneys, E. Doedel, W. Govaerts, Y. A. Kuznetsov, and B. Sandstede, Numerical continuation, and computation of normal forms, in Handbook of Dynamical Systems II,

Copyright (c) by SIAM. Unauthorized reproduction of this article is prohibited. 
Vol. 2, B. Fiedler, ed., Elsevier, Amsterdam, 2002, pp. 149-219.

[5] L. Bruno, A. Tosin, P. Tricerri, And F. Venuti, Non-local first-order modelling of crowd dynamics: A multidimensional framework with applications, Appl. Math. Model., 35 (2011), pp. 426-445.

[6] M. Di Francesco, P. Markowich, J.-F. Pietschmann, and M.-T. Wolfram, On the Hughes' model for pedestrian flow: The one-dimensional case, J. Differential Equations, 250 (2011), pp. 1334-1362.

[7] E. Doedel, H. Keller, ANd J. Kernevez, Numerical analysis and control of bifurcation problems. I. Bifurcation in finite dimensions, Internat. J. Bifur. Chaos Appl. Sci. Engrg., 1 (1991), pp. 493-520.

[8] E. Doedel, H. Keller, And J. Kernevez, Numerical analysis and control of bifurcation problems. II. Bifurcation in infinite dimensions, Internat. J. Bifur. Chaos Appl. Sci. Engrg., 1 (1991), pp. 745-772.

[9] W. E and B. Engquist, The heterogeneous multiscale methods, Commun. Math. Sci., 1 (2003), pp. 87132.

[10] V. A. Epanechnikov, Non-parametric estimation of a multivariate probability density, Theory Probab. Appl., 14 (1969), pp. 153-158.

[11] P. Gawronski and K. Kulakowski, Crowd dynamics-being stuck, Comput. Phys. Comm., 182 (2011), pp. 1924-1927.

[12] C. W. Gear, I. G. Kevrekidis, and C. Theodoropoulos, Coarse integration/bifurcation analysis via microscopic simulators: Micro-Galerkin methods, Comput. Chem. Eng., 26 (2002), pp. 941-963.

[13] J. Guckenheimer And P. Holmes, Nonlinear Oscillations, Dynamical Systems, and Bifurcations of Vector Fields, Appl. Math. Sci. 42, Springer-Verlag, New York, Berlin, Heidelberg, Tokyo, 1983.

[14] H. Haken, Advanced Synergetics, Springer Ser. Synergetics 20, Springer-Verlag, Heidelberg, Berlin, New York, 1983.

[15] H. Haken, Synergetics, An Introduction, Springer Ser. Synergetics 1, Springer-Verlag, Heidelberg, Berlin, New York, 1983.

[16] B. D. Hassard, N. D. Kazarinof, And Y.-H. Wan, Theory and Applications of Hopf Bifurcation, Cambridge University Press, Cambridge, UK, New York, 1981.

[17] D. Helbing, Traffic and related self-driven many-particle systems, Rev. Modern Phys., 73 (2001), pp. $1067-1140$.

[18] D. Helbing, I. Farkas, And T. Vicsek, Simulating dynamical features of escape panic, Nature, 407 (2000), pp. 487-490.

[19] D. Helbing, R. Jiang, And M. Treiber, Analytical investigation of oscillations in intersecting flows of pedestrian and vehicle traffic, Phys. Rev. E, 72 (2005), 046130.

[20] D. Helbing, J. Keltsch, And P. MolnáR, Modelling the evolution of human trail systems, Nature, 388 (1997), pp. 47-50.

[21] D. Helbing And P. Molnár, Social force model for pedestrian dynamics, Phys. Rev. E, 51 (1995), pp. $4282-4286$.

[22] D. J. Higham, An algorithmic introduction to numerical simulation of stochastic differential equations, SIAM Rev., 43 (2001), pp. 525-546.

[23] C. Huepe, G. Zschaler, A.-L. Do, And T. Gross, Adaptive-network models of swarm dynamics, New J. Phys., 13 (2011), 073022.

[24] R. Jiang, D. Helbing, P. K. Shukla, and Q.-S. Wu, Inefficient emergent oscillations in intersecting driven many-particle flows, Phys. A, 368 (2006), pp. 567-574.

[25] I. G. KeVREKIDIS, Equation-free, coarse-grained multiscale computation: Enabling microscopic simulators to perform system-level tasks, Commun. Math. Sci., 1 (2003), pp. 715-762.

[26] I. G. Kevrekidis And G. Samaey, Equation-free multiscale computation: Algorithms and applications, Ann. Rev. Phys. Chem., 60 (2009), pp. 321-344.

[27] Y. Kevrekidis and G. Samaey, Equation-free modeling, Scholarpedia, 5 (2010), p. 4847.

[28] H. Kitahata, A. Yamada, And S. Nakata, Mode bifurcation by pouring water into a cup, J. Chem. Phys., 119 (2003), pp. 4811-4816.

[29] M. I. Kohira, N. Magome, H. Kitahata, and K. Yoshikawa, Plastic bottle oscillator: Rhythmicity and mode bifurcation of fluid flow, Amer. J. Phys., 75 (2007), pp. 893-895.

[30] M. I. Kohira, N. Magome, S.-I. Mouri, H. Kitahata, and K. Yoshikawa, Synchronization of three coupled plastic bottle oscillators, Int. J. Unconventional Comput., 5 (2009), pp. 103-111.

[31] T. Kretz, M. WÖLKI, And M. Schreckenberg, Characterizing correlations of flow oscillations at bottlenecks, J. Stat. Mech., February (2006), P02005.

Copyright (C) by SIAM. Unauthorized reproduction of this article is prohibited. 
[32] J. LegA, Collective behaviors in two-dimensional systems of interacting particles, SIAM J. Appl. Dyn. Syst., 10 (2011), pp. 1213-1231.

[33] J. E. Marsden And M. McCracken, The Hopf Bifurcation and Its Applications, Springer-Verlag, New York, 1976.

[34] MATLAB, Version 7.9.0.529 (R2009b), http://www.mathworks.com, 2009.

[35] P. Molnár and J. Starke, Control of distributed autonomous robotic systems using principles of pattern formation in nature and pedestrian behaviour, IEEE Trans. Syst. Man Cybern. B Cybern., 31 (2001), pp. 433-436.

[36] M. Moussaid, D. Helbing, and G. Theraulaz, How simple rules determine pedestrian behavior and crowd disasters, Proc. Natl. Acad. Sci. USA, 108 (2011), pp. 6884-6888.

[37] S. Nakata, A. Yamada, AND H. Kitahata, Mode-bifurcation upon pouring water into a cup that depends on the shape of the cup, Phys. Lett. A, 339 (2005), pp. 45-51.

[38] G. Nicolis and I. Prigogine, Self-Organization in Non-equilibrium Systems, Wiley, New York, 1977.

[39] S. Paris, J. Pettré, And S. Donikian, Pedestrian reactive navigation for crowd simulation: A predictive approach, Eurographics, 26 (2007), pp. 665-674.

[40] W. Press, S. Teukolsky, W. Vetterling, and B. Flannery, Numerical Recipes in C, Cambridge University Press, Cambridge, UK, New York, 1992.

[41] H. Spohn, Large Scale Dynamics of Interacting Particles, Springer-Verlag, Berlin, 1991.

[42] J. Starke, C. Ellsässer, And T. Fukuda, Self-organized control in cooperative robots using a pattern formation principle, Phys. Lett. A, 375 (2011), pp. 2094-2098.

[43] C. Theodoropoulos, Y.-H. Qian, And I. G. Kevrekidis, Coarse stability and bifurcation analysis using time-steppers: A reaction-diffusion example, Proc. Natl. Acad. Sci. USA, 97 (2000), pp. 9840 9843.

Copyright $\odot$ by SIAM. Unauthorized reproduction of this article is prohibited. 